\title{
Cobweb Chaos in Agricultural Markets:
}

\author{
Simulation Results
}

Akio Matsumoto*

\begin{abstract}
This study augments the traditional linear cobweb model with lower and upper bounds for variations of output. Its purpose is to detect the relationship between the output constraints and the dynamics of the modified model. Due to the upper and lower bounds, a transitional function takes on a tilted $z$-profile having three piecewise segments with two turning points. It prevents the price (or quantity) dynamics from explosive oscillations. This study demonstrate, by presenting numerical examples, that the modified cobweb model can generate various dynamics ranging from stable periodic cycles to ergodic chaos if a product of the marginal propensity to consume and the marginal product is greater than unity.
\end{abstract}

\section{Introduction}

This study investigates the traditional cobweb model with upper and lower bounds for output variations. Its purpose is to consider implications of the quantity constraints on dynamic behavior of the agricultural economy. The traditional cobweb model, which has naive expectations and linear demand and supply curves, can produce only three types of dynamics: convergence to an equilibrium, convergence to period-2 cycles or divergence. None of these types, however, is satisfactory to explain observed irregular fluctuations of the agricultural goods. Neither the first type nor the third is consistent with observed ups and downs in real economic data, and the second, which implies persistent oscillations in price and quantity, depends on the unrealistic and shaky condition (i.e., supply and demand have exactly the same elasticity). To overcome those limitations, the traditional cobweb model has been modified to produce more realistic dynamics with the help of new developments in non-linear economic dynamics.

Modifications fall into two groups. In the first of these, I have endogenous non-linear cobweb models in which the supply and/or demand curve are non-linear. The resulting

\footnotetext{
* Niigata University Department of Economics, 8050, Ni-No-Cho, Ikarashi, Niigata 950-21, Japan. Tel : +81/(0)25-262-6551, Fax: +81/(0)25-263-3262

E-mail : eakio@hle. niigata-u.ac.jp

I would like to thank Y. Sakai, A. Simonovits, K. Higuchi and two anonymous referees for valuable comments. The earlier version of the paper also benefited from comments received during presentations at the international conference on "Commerce, Complexity and Evolution" at University of New South Wales, Sydney, Australia, February 12-13, 1996 and the annual meeting of Japan Regional Science Association at Rissho University, Tokyo, October 7-8, 1995. However, all remaining errors are my own.
} 
transition functions are chaotic maps having either one turning point or two. Several stability results have been established, which show chaotic oscillations as well as stable periodic cycles (see, Jensen and Urban [12], Chiarella [2], Day and Hanson [4], Finkenstädt $[7]$, Hommes $[8,9]$ ). In the second, I find the linear cobweb model taking account of upper bound for variations of output. The upper bound prevents the price (or quantity) dynamics from explosive oscillations. The resulting one-dimensional control map is similar to an expansive tent map (i.e., its slopes are steeper than unity in absolute value). Consequently, it can be shown that the linear cobweb model with upper bound generates not only topological (Li-Yorke) chaos but also ergodic chaos (see Cugno and Montrucchio [1], Nusse and Hommes [15]). This model, however, has a possibility that almost all trajectories escape from an economically meaningful region for some parameter constellations. In such a case, trajectories are unbounded and thus it is unable to track the price-quantity evolution.

The study extends the second approach. Returning to the original spirits of "flexible constraints" in Day [3], it constructs the linear cobweb model augmented with the lower bound for variations of output as well as the upper bound. Its purpose is to detect the relationship between the output constraints and the dynamics of the model. In particular, by presenting numerical examples, it demonstrates that the modified cobweb model can generate a wide spectrum of dynamic behavior ranging from stable periodic cycles to ergodic chaos.

This study is organized as follows. Section 2 constructs the linear cobweb model with upper and lower bounds for output variations. Section 3 simulates the model. Section 4 makes concluding remarks.

\section{The Cobweb Model with Flexible Constraints}

The traditional cobweb model in discrete time is made up of the following four equations :

$$
\begin{aligned}
& q_{t}^{d}=D\left(p_{t}\right), \text { Demand, } \\
& q_{t}^{s}=S\left(p_{t}^{e}\right), \text { Supply, } \\
& q_{t}=q_{t}^{d}=q_{t}^{s}, \text { Temporary Equilibrium, } \\
& p_{t}^{e}=p_{t-1}, \quad \text { Naive Expectation. }
\end{aligned}
$$

This model can be reduced to a one-dimensional difference equation:

$$
q_{t+1}=S\left(D^{-1}\left(q_{t}\right)\right) \text {. }
$$

In a simple version of the traditional cobweb model, the demand function as well as the supply function is monotonic and thus the composite map, $S\left(D^{-1}\left(q_{t}\right)\right)$, is also monotonic. The slope of $S\left(D^{-1}\left(q_{t}\right)\right)$ evaluated at the equilibrium point characterizes dynamics, since it equals an eigenvalue of the dynamic equation. As long as the slope lies between 0 and $-45^{\circ}$, the eigenvalue in absolute value is less than unity, and thus the equilibrium point is stable. The stable trajectories of price or quantity converge to a stationary state, which does not go with the price or quantity dynamics observed in the real-world. As the slope steepens beyond $-45^{\circ}$, the eigenvalue in absolute value is greater than unity and thus the equilibrium point is unstable. The unstable trajectories explosively oscillate, which also contradicts the actual dynamic behavior. When the slope is equal to $-45^{\circ}$, period -2 cycles can appear. 


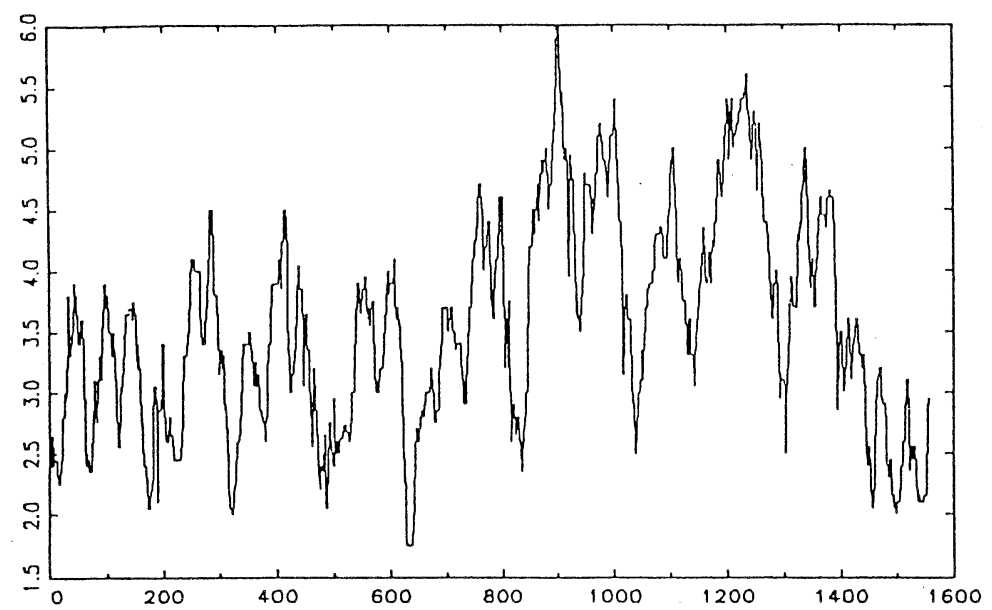

Fig. 1(a) Weekly pig prices from 1958 to 1988 (this is an upper half of Figure 3.1 in Finkenstädt $[7$, p. 66]).

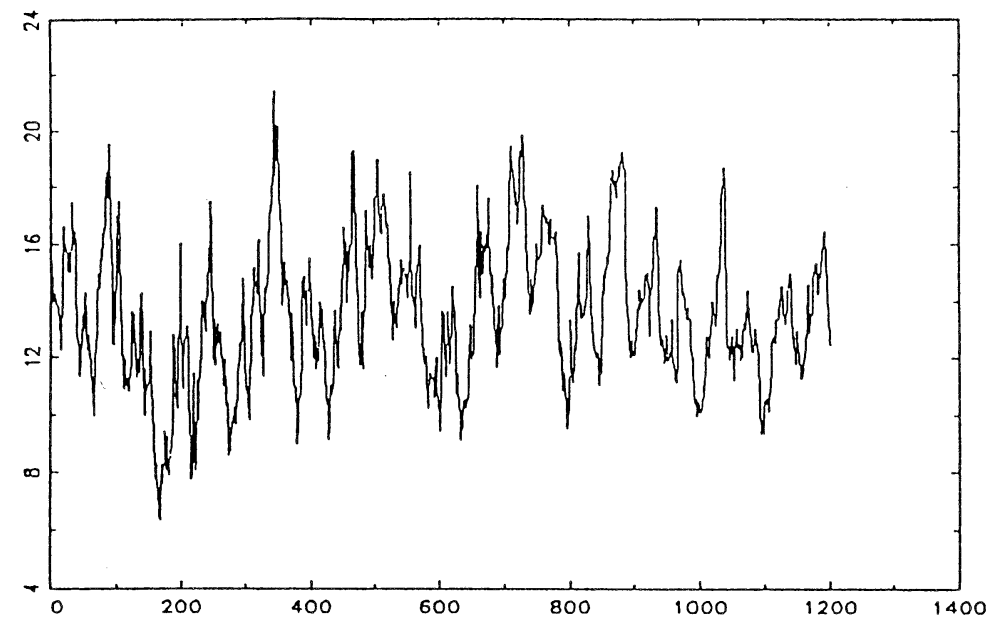

Fig. 1(b) Weekly egg prices from 1967 to 1990 (this is an upper half of Figure 3.3 in Finkenstädt $[7$, p. 68]).

However, the regular cycles are unlike the irregura nature of the actual cycles. Thus such a simple cobweb model has difficulties to explain erratic movements observed in statistical data of agricultural goods. In Figures 1(a) and (b), it is seen that agricultural prices behave fairly volatile.

To overcome those difficulties, Cugno and Montrucchio [1], who retain the linear specifications of supply and demand, modify the simple cobweb model by introducing the upper constraint on $q_{t}$ :

$$
\begin{aligned}
S\left(p_{t-1}\right) & =-a+b p_{t-1}, a>0, b>0, \\
D^{-1}\left(q_{t}\right) & =c-d q_{t}, c>0, d>0, \\
q_{t+1} \leq q_{t+1}^{U} & =(1+\alpha) q_{t}, \alpha>0 .
\end{aligned}
$$


The last equation implies that the upper constraint has the effect of preventing output in period $t+1$ from increasing by more than $100 \alpha \%$ from the output of period $t$. Alternatively put, $\alpha$ is an upper bound imposed on the growth rate of output (i.e., $\frac{q_{t+1}-q_{t}}{q_{t}} \leq \alpha$ ). The resultant dynamic equation is unimodal piecewise linear:

$$
q_{t+1}=\max \left\{(1+\alpha) q_{t}, b c-a-b d q_{t}\right\},
$$

which take the extremum at $\bar{q}$ for some $\bar{q} \in\left[0, \frac{b c-a}{b d}\right]$ and is linear on each of two subintervals, $[0, \bar{q}]$ and $\left[\bar{q}, \frac{b c-a}{b d}\right]$. Cugno and Montrucchio [1] focus on a unstable case in which $b d>1$ and demonstrate that if the following inequality conditions are satisfied, the cobweb model with upper the output constraint can yield dynamics resemble to realistic (i.e., chaotic) behavior:

$$
\frac{1+\alpha}{\alpha}>b d \geq \frac{2+\alpha}{1+\alpha}
$$

where the first inequality is a condition for trajectories to be bounded and the second inequality is necessary for the appearance of a period-3 cycle. Thus dynamic equation (2) that satisfies the above inequality conditions generates persist and irregular motions.

Utilizing the fact that the dynamic equation, (2), is expansive (i.e., the slope steepens greater than either $45^{\circ}$ or $-45^{\circ}$ ), Nusse and Hommes [15] widen the Cugno and Montrucchio condition and obtain:

$$
\frac{1+\alpha}{\alpha} \geq b d>1
$$

They demonstrate that both the 'Li-Yorke' chaos and the 'sensitive dependence on initial values' chaos can occur in the modified cobweb model. ${ }^{1}$ In a case $b d>\frac{1+\alpha}{\alpha}$, almost all (with respect to the Lebesgue measure) trajectories eventually escape from a trapping interval, $\left[0, \frac{b c-a}{b d}\right]$, and either fall to zero if non-negative constraint is imposed or go to negative infinity if not.

To get rid of such economically unrealistic behavior, I modify the Cugno-Montrucchio cobweb model. Returning to the original spirits of "flexible coefficient" in Day [3], ${ }^{2}$ I add a lower bound constraint on output :

$$
q_{t+1} \geq q_{t}^{L}=(1-\beta) q_{t-1}, 1>\beta>0 .
$$

This constraint, analogous to the upper bound constraint, have the effect of preventing output in period $t$ from decreasing by more than $100 \beta^{0} \%$ from the output of period $t-1$. Alternatively put, $\beta$ is a lower bound imposed on the (negative) growth rate (i.e., $\frac{q_{t+1}-q_{t}}{q_{t}} \geq-\beta$ ). The lower and upper bound constraints work to compress trajectories into a cone spanned by $q_{t+1}^{U}=(1+\alpha) q_{t}$ and $q_{t}^{L}=(1-\beta) q_{t-1}$ in the phase space. Since in a real world it faces the

\footnotetext{
1 The dynamic equation (2) that satisfies the last inequality conditions has an absolutely continuous measure. See Theorem 3 of Day and Pianigiani [5].

2 See Day [3, p. 197] who considers the symmetric upper and lower constraints.
} 
capacity constraint as well as the financial constraint and cautiously responses to the extremely fluctuating prices, it is not surprising that the a competitive flrm limits the output growth rate. ${ }^{3}$

The linear cobweb model with the upper and lower bound constraints has the following piecewise linear map that governs the asymptotic behavior of $q_{t}$ :

$$
f\left(q_{t}\right)= \begin{cases}(1+\alpha) q_{t} & \text { for } q_{t} \leq Q_{M}, \\ b c-a-d b q_{t} & \text { for } Q_{M} \leq q_{t} \leq Q_{m}, \\ (1-\beta) q_{t} & \text { for } q_{t} \geq Q_{m},\end{cases}
$$

where a maximizer $Q_{M}$ and a minimizer $Q_{m}$ are calculated, respectively, as

$$
Q_{M}=\frac{b c-a}{1+\alpha+b d} \text { and } Q_{m}=\frac{b c-a}{1-\beta-b d} .
$$

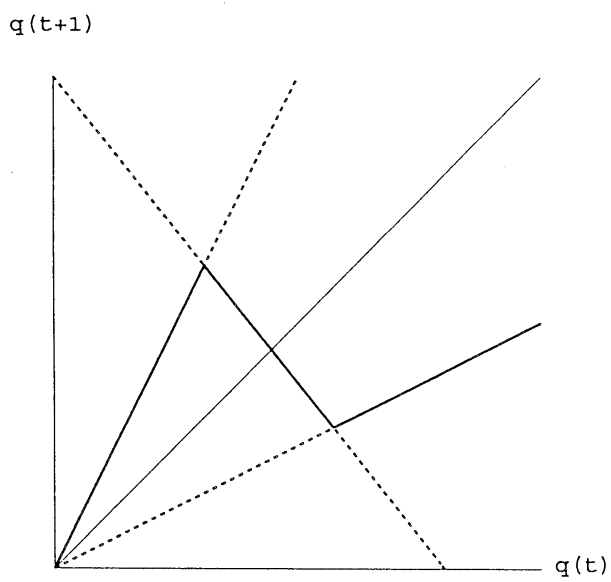

Fig. 2(a) $(1+\alpha)(1-\beta)=1$.

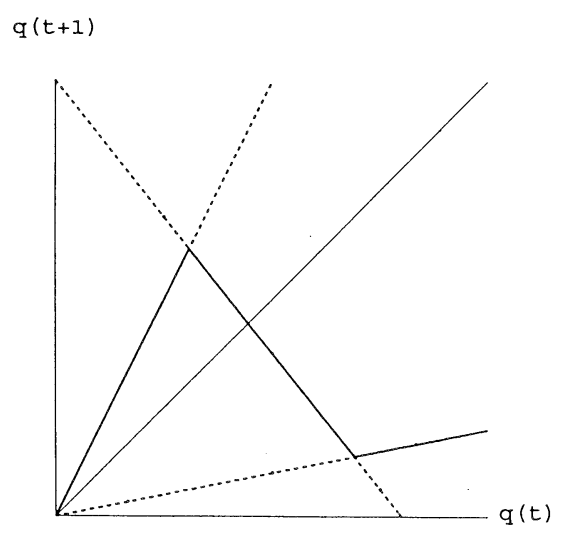

Fig. 2(b) $\quad(1+\alpha)(1-\beta)<1$.

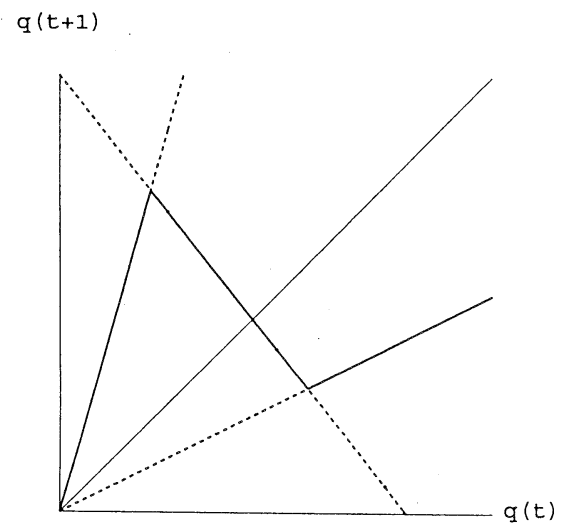

Fig. 2(c) $(1+\alpha)(1-\beta)>1$.

\footnotetext{
${ }^{3}$ Such a cautious response of a firm is pointed out by Huang [10].
} 
For $b d>0, b c-\alpha>0, \alpha>0$, and $1>\beta>0$, the map has the tilted- $z$ profile and its non-linearity becomes more pronounced as $b d$ gets larger.

Let $q^{*}$ be an equilibrium output satisfying $q^{*}=f\left(q^{*}\right)\left(\right.$ i.e., $\left.q^{*}=\frac{b c-a}{1+b d}\right)$. If $b d>1$, it is oscillatory unstable and forces trajectories to move away in its neighborhood. But fluctuations are bounded due to the upper and lower constraints of output and thus perpetuated. In order to explain the limiting behavior of bounded fluctuations, I identify three cases that depend on the relative magnitudes between $\alpha$ and $\beta$ : (1) $(1+\alpha)(1-\beta)=1 ;(2)(1+\alpha)(1-\beta)<$ $1 ;(3)(1+\alpha)(1-\beta)>1$. The correspondings profles of $f\left(q_{t}\right)$ are depicted, respectively, in Figures 2(a), 2(b), and 2(c) where a cone spanned by the upper and lower constraints is symmetric with respect to $45^{\circ}$ line in the first case and asymmetric in the second and third cases. Considerable progress is found in the study on a map with two piecewise linear segments and a single kinked point like a tent map. ${ }^{4}$ However, only the partial results are obtained in the study on a map with three piecewise linear segments and two kinked points like equation (3). ${ }^{5}$ Thus, by simulating the model under different values of $b d(>1)$, I can investigate the dynamic structure of the modified cobweb model under each of these three parameter constellations for $\alpha$ and $\beta$.

\section{Simulation of the Model}

To explore the dynamic behavior of $q$, I simulate the model under different values of $b d$ were $b$ is the marginal propensity to consume and $d$ is the marginal product with respect to the price. I focus on those cases in which bounded oscillations are perisistent (i.e., $b d>1$ ). Figures 3-5 below are bifurcation diagrams in which a bifurcation parameter is $b d, b c-a=$ 10 , and the upper and lower bounds for the growth rate of output, $\alpha$ and $\beta$, are fixed at various values : $\alpha=1, \beta=0.5$, in Figures $3 ; \alpha=2, \beta=0.8$, in Figure $4 ; \alpha=1 \beta=0.25$, in Figure 5 .

\subsection{Symmetric case}

The first simulation is shown in Figure 3(a) and is performed under the symmetric constraints condition, $(1+\alpha)(1-\beta)=1$, and an initial condition, $q_{0}=0.1$. The parameter $\frac{1}{b d}$ is varied in decrement of 0.025 from one to zero. For each values of $\frac{1}{b d}, f\left(q_{t}\right)$ is iterated 200 times. Although the last 100 iterates are plotted on the vertical axis, only two points are shown in the bifurcation diagram. This suggests that the symmetrically constrained cobweb model generate only period-2 cycles. This result is verified as follows. I denote a local minimum by $Q^{\min }:=f\left(Q_{m}\right)$ and a local maximum by $Q^{\operatorname{Max}}:=f\left(Q_{M}\right)$, both of which depend on $b d$ as $Q_{m}$ and $Q_{M}$ do. Let $A_{0}:=\left[Q^{\min }, Q_{M}\right]$ and $A_{1}:=\left[Q_{m}, Q^{\operatorname{Max}}\right]$ where $Q^{\min }<Q_{M}<Q_{m}<$ $Q^{\text {Max }}$ holds in the symmetric case. Then it can be checked that $f\left(A_{0}\right)=A_{1}, f\left(A_{1}\right)=A_{0}$, and a restriction of $f^{2}(q)=f(f(q))$ to $A_{i}$ is the identity map on $A_{i}(i=0,1)$. Every trajectory emanating form an interval $\overline{A_{0} \cup A_{1} \cup\left\{q^{*}\right\}}$ enter into $A_{0} \cup A_{1}$. Thus I have stable period-2 cycles in the symmetric case.

Depending on initial points, there are three kinds of period-2 cycle. I call a period-2

\footnotetext{
${ }^{4}$ See Day and Pianigiani [5].

${ }^{5}$ See Day and Shafer [6] and Matsumoto [13].
} 
cycle upper-constrained if it is bounded only by the upper constraint, lower-constrained if only by the lower constraint or both-constrained if by the upper and lower constraints. An orbit $\left\{Q_{m}, Q^{\mathrm{min}}\right\}$ is the upper-constrained cycle since it is a period-2 iteration, $Q^{\mathrm{min}}=(1-\beta) Q_{m}$, $Q_{m}=(1+\alpha) Q^{\min }$, and the upper constraint is effective for $Q^{\min },(1+\alpha) Q^{\min }<S\left(D^{-1}\left(Q^{\min }\right)\right)$. An orbit $\left\{Q_{M}, Q^{\mathrm{Max}}\right\}$ is the lower-constrained cycle since it is a period-2 iteration, $Q^{\mathrm{Max}}=(1+$ $\alpha) Q_{M}, Q_{M}=(1-\beta) Q^{\operatorname{Max}}$ and the lower constraint is effective for $Q^{\operatorname{Max}},(1-\alpha) Q^{\operatorname{Max}}>$ $S\left(D^{-1}\left(Q^{\text {Max }}\right)\right)$. Any other pair of period-two iterations, $\left\{q_{t}, f\left(q_{t}\right)\right\}$ where $q_{t}$ as well as $f\left(q_{t}\right)$ is an interior point of $A_{0}$ or $A_{1}$, is a both-constrained cycle as it satisfies $(1+\alpha) q_{t}=f\left(q_{t}\right)$ and $(1-\beta) f\left(q_{t}\right)=q_{t}$. In Figure 3(b), two bifurcation diagrams, one for the upper-constrained cycle (solid curves) and the other for the lower-constrained cycle (dot curves) are superimposed on the bifurcation diagram of Figure 3(a) (i.e., the one for the upper- and lowerconstrained cycle). It can be observed that the amplitude of the upper-or lower-constrained
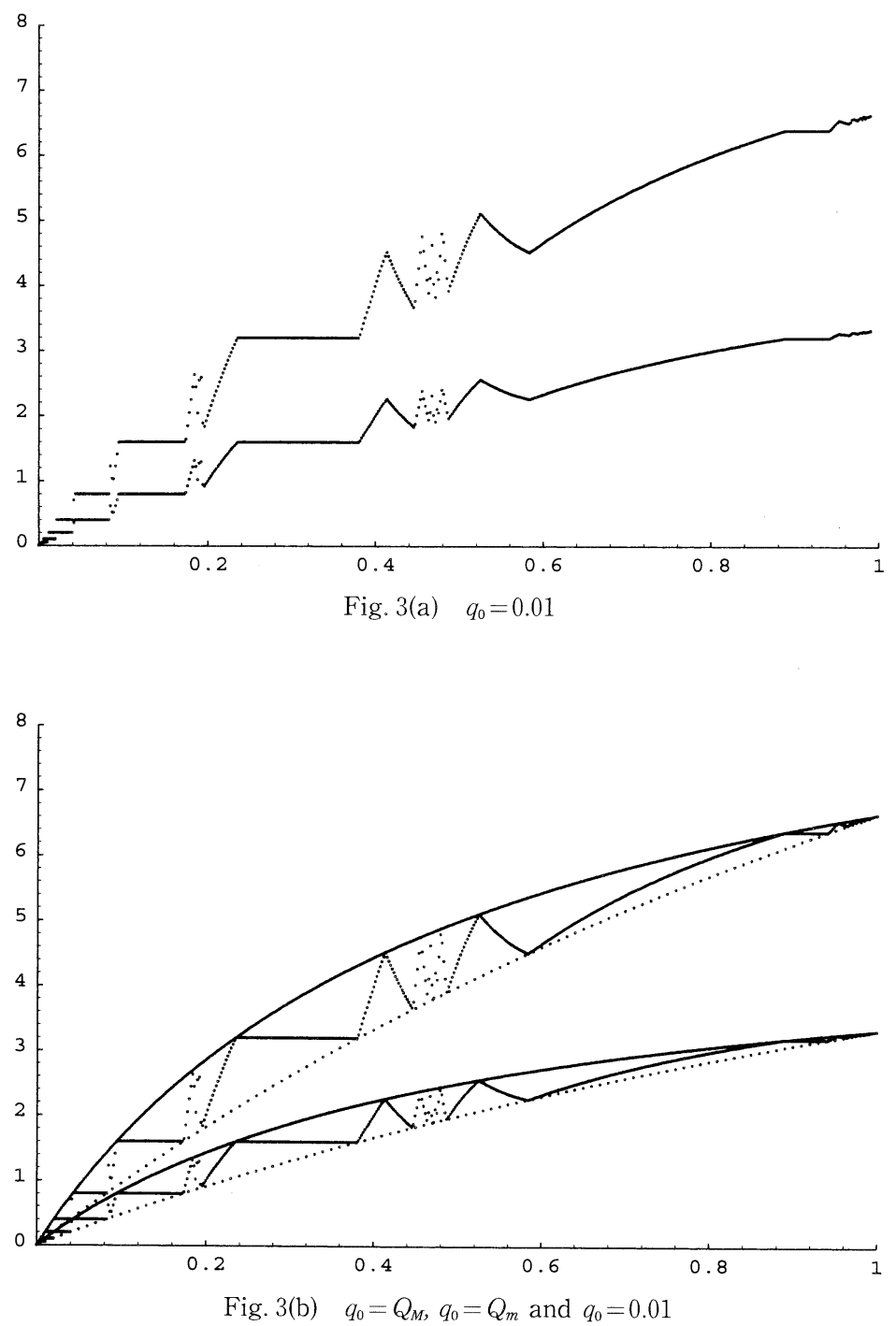
cycle is monotonically decreasing in $b d$ and that although fluctuations in the amplitude of the both-constrained cycle are viable, these are bounded from below by the upper-constrained cycle and from above by the lower-constrained cycle.

Furthermore, applying the profit analysis to the symmetric case, I can show that there is a case in which the period- 2 cycle is profitable to a competitive equilibrium if it is close to the upper-constrained cycle and unprofitable if it is close to the lower-constrained cycle. ${ }^{6}$

\subsection{Lower asymmetric case}

The second simulation is shown in Figure 4 and is performed under the asymmetric constraints condition $(1+\alpha)(1-\beta)<1$. As seen in Figure $1(b)$, the lower constraint line deviates from the $45^{\circ}$ line more than the upper constraint line so that I call this case lower asymmetric. Since the dynamic equation, $f(q)$, has the upper and lower bounds, it induces any trajectories, which are repelled by the unstable equilibrium, bounce back to a neighborhood of the equilibrium point. Thus I can define a trapping interval by an interval that eventually traps all trajectories. A restriction of $f(q)$ to the trapping interval governs the asymptotic behavior of $q$. Two distinct cases can be identified which depending on the relation between miximum $Q^{\max }$ and minimizer $Q_{m}$ : one where $Q^{\max }<Q_{m}$; the other where $Q^{\max }>Q_{m}$. Since the lower asymmetric constraints condition, $(1+\alpha)(1-\beta)<1$, is written as $\frac{\alpha}{1+\alpha}<\beta$, there is a value of $b d$, denoted by $(b d)^{L}$, such that $(b d)^{L} \frac{\alpha}{1+\alpha}=\beta$. It can be checked that $Q^{\max } \leq Q_{m}$ holds for $1<b d \leq(b d)^{L}$ and $Q^{\max }>Q_{m}$ for $b d>(b d)^{L}$.

Let $V_{1}:=\left[f\left(Q^{\max }\right), Q^{\max }\right]$ where $f\left(Q^{\max }\right)=b c-a-b d Q^{\max }$. Then it becomes the trapping interval for $1<b d \leq(b d)^{*}$. The restriction of $f\left(q_{t}\right)$ to $V_{1}$ is essentially the same as equation (2). That is, it is expansive so that every cycle is unstable and there exists an absolutely continuous invariant measure. In consequence, it can generate complex dynamics including ergodic chaos. ${ }^{7} \quad$ Let $V_{2}:=\left[Q^{\min }, Q^{\max }\right]$. Then it becomes the trapping interval for $b d>(b d)^{L}$. Since it contains two kinked points, $Q_{M}$ and $Q_{m}$, a restriction of $f\left(q_{t}\right)$ to $V_{2}$ takes

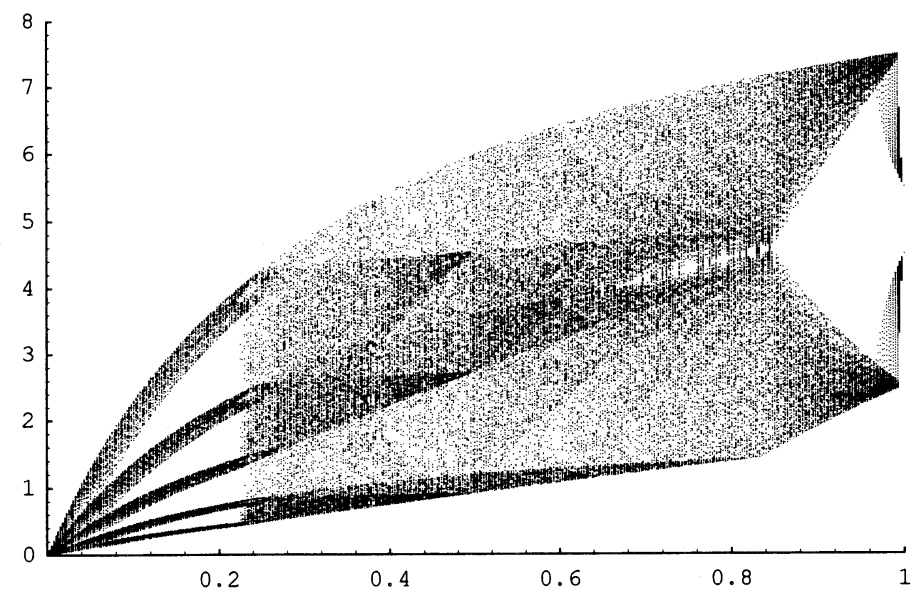

Fig. 4

${ }^{6}$ See Huang [10] and Matsumoto [14]. 
on a tilted $z$-shape:

$$
f_{\mid V_{2}}\left(q_{t}\right)=\max \left\{(1-\beta) q_{t}, \min \left\{(1+\alpha) q_{t}, b c-a-b d q_{t}\right\}\right\} .
$$

Day and Schafer [1987, Table 1 at p. 355] make some characterization for such a map with two turning points. Figure 4 suggests that chaotic behavior appears as $b d$ is increased from $(b d)^{L}{ }^{8}$ If $b d$ is further increased, noisy chaos with five intervals appears, that is, trajectories periodically visit one interval to another but aperiodically oscillate within an interval.

\subsection{Upper asymmetric case}

The third simulation is shown in Figure 5 and is performed under the asymmetric constraint condition, $(1+\alpha)(1-\beta)>1$. The analysis in this subsection is similar to that in the last subsection although a direction of the equality in the constraint condition is opposite. As seen in Figure 1(c), the upper constraint line deviates from the $45^{\circ}$ line more than the lower constraint line so that I call this case upper asymmetric. Similar to the lower asymmetric case, I can identify two cases, depending on the relation between minimum $Q^{\min }$ and maximizer $Q_{M}$ : one where $Q_{M}<Q^{\min }$ : the other where $Q_{M}>Q^{\min }$. Since the upper constraint condition, $(1+\alpha)(1-\beta)>1$, is written as $\frac{\beta}{1-\beta}<\alpha$, there is a value of $b d$, denoted by $(b d)^{U}$, such that $(b d)^{U} \frac{\beta}{1-\beta}=\alpha$. It can be checked that $Q_{M} \leq Q^{\min }$ for $1<b d \leq(b d)^{U}$, and $Q_{M}>Q^{\min }$ for $b d>(b d)^{U}$.

Let $U_{1}:=\left[Q^{\min }, f\left(Q^{\min }\right)\right]$ where $f\left(Q^{\min }\right)=b c-a-b d Q^{\min }$. Then it is a trapping interval for $1<b d \leq(b d)^{U}$. A restricted of $f(q)$ to $U_{1}$,

$$
f_{\mid U_{1}}(q)=\max \left\{b c-a-b d q_{t},(1-\beta) q_{t}\right\},
$$

governs asymptotic dynamics of $q_{t}$. I call $f_{\mid U_{1}}(q)$ an asynmmetric tent map as it has a slope

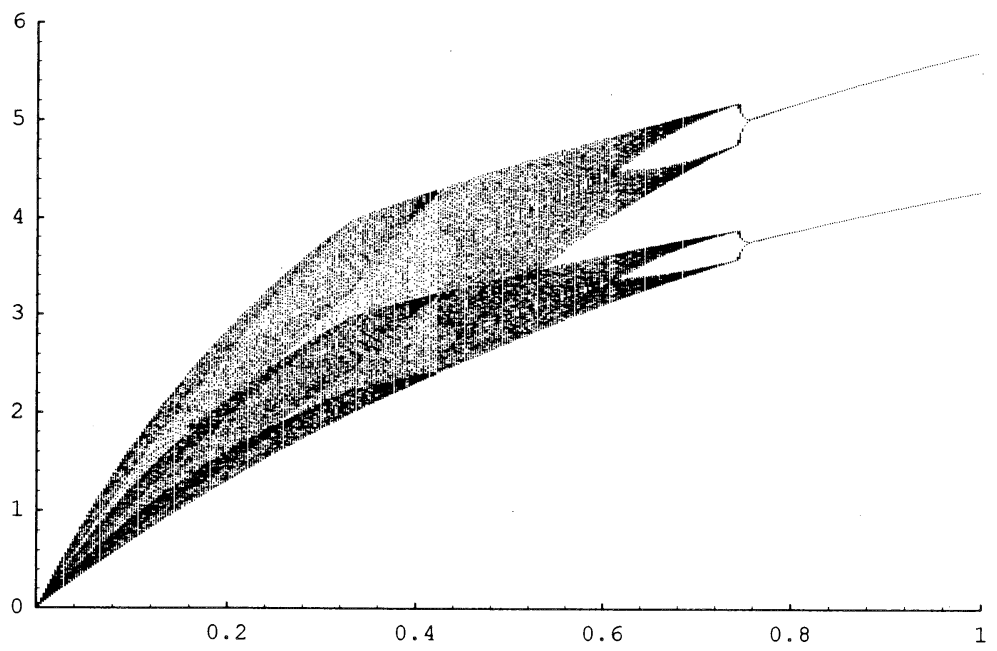

Fig. 5

${ }^{7}$ See Theorem 3 of Day and Schafer [6, pp. 352-3], (3) in Property 5 of Nusse and Hommes [15, p. 13] and Appendix of Matsumoto [14].

${ }^{8}$ Since $\alpha=2$ and $\beta=0.8$ is assumed, $(b d)^{\mathrm{L}} \simeq 0.83$. 
greater than unity in absolute value (i.e., $\left|\frac{\partial f_{\mid U_{1}}(q)}{\partial q}\right|=b d>1$ ) on a subinterval, $\left[Q^{\min }, Q_{m}\right)$ and one less than unity (i.e., $\left.\frac{\partial f_{1} U_{1}(q)}{\partial q}=1-\beta<1\right)$ on a subinterval, $\left(Q_{m}, f\left(Q^{\min }\right)\right]$. It is not expansive. Thus neither the Nusse-Hommes results nor the Day-Schafer results can be used in the upper asymmetric case. However, $f_{\mid U_{1}}(q)$ can be shown to be linearly conjugate to a unimodal linear map with two parameters $A$ and $B^{9}$,

$$
g_{A, B}(q)= \begin{cases}A q+\frac{A+B-A B}{B} & \text { for } 0 \leq q \leq 1-\frac{1}{B}, \\ -B(q-1) & \text { for } 1-\frac{1}{B} \leq q \leq 1 .\end{cases}
$$

That is, $f_{\mid U_{1}}(q)$ generates qualitatively the same dynamics as $g_{A, B}(q)$. Ito, Tanaka, and Nakada [11] give a complete characterization of such unimodal linear transformation. Hence, applying their results (i.e., Corollary 3.1 or Theorem 3.1), I can show that the restricted map, $f_{\mid U_{1}}$, has an absolutely continuous invariant ergodic measure. Figure 5 suggests the following bifurcation scenario. If $b d$ is increased from unity, the trajectory is under the influence of the asymmetric tent map. The equilibrium point bifurcates to stable period- 2 cycles, noisy chaos with $2^{2}$ intervals, 2 intervals, and 1 interval (i.e., truly chaos). If $b d$ is further increased from $(b d)^{U}, Q^{\min }$ becomes less than $Q$. Then the dynamic system is switched from (5) to a restriction of $f\left(q_{t}\right)$ to a trapping interval $U_{2}:=\left[Q^{\min }, Q^{\max }\right]$ having two kinked points, $Q_{M}$ and $Q_{m}$. The tilted $z$-shaped restricted map governs the quantity evolution and, according to Figure 5, generates aperiodic fluctuations.

\section{Concluding Remarks}

This study investigates the dynamic structure of the linear cobweb model with upper and lower bounds for variations on output. Simulating the model under different values of $b d$, I have demonstrated that the modified cobweb model may generate chaotic behavior if the output constraints are asymmetric and that it can generate stable period- 2 cycle whose amplitudes depend on the prevailing parameter constellations (i.e., choice of initial point, values of $b d$, etc.) if the output constraints are symmetric. Although the bifurcation diagrams in asymmetric cases imply the chaotic behavior, the bifurcation scenario to chaos is different according to whether the constraints are upper or lower asymmetric. In the lower symmetric case, the modified cobweb model generates persistent and aperiodic cycles for any values of $b d$ greater than unity. The control system (i.e., a restriction of $f(q)$ to a trapping interval) switches from a expansive map to a tilted $z$-shaped map as $b d$ is increased. In consequence, chaos with two intervals and then truly chaos appear as $b d$ exceeds unity. Noisy chaos with 5 intervals appears if $b d$ is further increased. On the other hand, in the upper asymmetric case, the control system switches form a asymmetric tend map to a tilted $z$-shaped map. A road to chaos in the upper symmetric case is different form on in the lower symmetric case. As $b d$ is increased from unity, the quantity dynamics (as well as the price dynamics) move from stable period- 2 cycle, to noisy chaos with $2^{2}$ intervals, to chaos with 2 intervals and then to truly chaos (i.e., chaos with 1 interval), the last of which shrinks to zero

\footnotetext{
${ }^{9}$ See Matsumoto [14].
} 
as $b d$ goes to infinity.

What implications do the simulations have for the cobweb model with the upper and lower bounds. $\quad b d>1$ is necessary for generations of chaos in the asymmetric case. Here $b$ is the marginal propensity to consume which is the decision variables of the demand side while $d$ is the marginal product with respect to price which is the decision variable of the supply side. Thus it can be stated that one source of such complex dynamics involving chaos is an interaction between the consumers and the producers. Furthermore, since the marginal propensity to consume is expected to satisfy condition $0<b<1, b d>1$ requires the strong production response to a change in price (i.e., the large value of $b$ ). The simulations, therefore, imply that the agricultural economy with the strong production response may exhibit erratic dynamics of the price and the agricultural goods.

\section{References}

[1] Cugno, F. and L. Montrucchio, "Some New Techniques for Modeling Nonlinear Economic Fluctuations: A Brief Survey," in R.M. Goodwin, M. Krüger, and A. Vercelli. eds., Nonlinear Models of Fluctuating Growth, Lecture Note in Economics and Mathematical System 228, Springer Verlag, Berlin, 1980, 1980, 146-165.

[2] Chiarella, C., "The Cobweb Model. Its Instability and the onset of Chaos," Economic Modeling 5, 1988, 1980, 377-384.

[ 3 ] Day, R., "Cobweb Models with Explicit Suboptimization," in R. Day and A. Cigno. eds., Modeling Economic Change : the Recursive Programming Approach, North Holland, Amsterdam, 1980, 191215.

[ 4 ] Day, R. and K. Hanson, "Cobweb Chaos," in T. Kaul and J. Sengupta eds., Essay in Honor of Karl A. Fox, Elsevier Science Publishers B.V., 1991, 175-192.

[5 ] Day, R. and R. Pianigiani, "Statistical Dynamics and Economics," Journal of Economic Behavior and Organization, 16, 1991, 37-83.

[6] Day, R. and W. Schafer, "Ergodic Fluctuations in Deterministic Economic Model," Journal of Economic Behavior and Organization 8, 1987, 339-361.

[ 7 ] Finkenstädt, B., Nonlinear Dynamics in Economics, Lecture Note in Economics and Mathematical System 426, Spring-Verlag, Berlin, 1994.

[ 8 ] Hommes, C., "Adaptive Learning and Roads to Chaos," Economic Letters 36, 1991, 127-132.

[ 9 ] Hommes, C., "Dynamic of the Cobweb Model with Adaptive Expectations and Nonlinear Supply and Demand," Journal of Economic Behavior and Organization 24, 1994, 315-335.

[10] Huang, W., "Caution Implies Profit," Journal of Economic Behavior and Organization 27, 1995, 257-277.

[11] Ito, S., Tanaka, S., and H. Nakada, "On Unimodal Linear Transformation and Chaos II," Tokyo Journal of Mathematics 2, 1979, 241-259.

[12] Jensen, R. and R. Urban, "Chaotic Price Behavior in a Non-Linear Cobweb Model, " Economic Letters 15, 1984, 235-240.

[13] Matsumoto, A., "Ergodic Chaos in Inventory Oscillations; An Example," forthcoming in Chaos, Solitions and Fractals, 1996.

[14] Matsumoto, A., "Ergodic Cobweb Chaos: Theory and Emprics," WEA International Annual Conference, San Francisco, USA June 28-July 2, 1996.

[15] Nusse, H. and C. Hommes, "Resolution of Chaos with Application to a Modified Samuelson Model," Journal of Economic Dynamics and Control 14, 1990, 1-19. 